\title{
ROBUST CONTROL FOR AN UNCERTAIN CHEMOSTAT MODEL
}

\author{
JEAN-LUC GOUZE AND GONZALO ROBLEDO
}

\begin{abstract}
In this paper we consider a control problem for an uncertain chemostat model with a general growth function and cell mortality. This uncertainty affects the model (growth function) as well as the outputs (measurements of substrate). Despite this lack of information, an upper bound and a lower bound for those uncertainties are assumed to be known a priori. We build a family of feedback control laws on the dilution rate, giving a guaranteed estimation on the unmeasured variable (biomass), and stabilizing the two variables in a rectangular set, around a reference value of the substrate. We give two realistic applications of this control law to a depollution process and to phytoplankton culture.
\end{abstract}

\section{INTRODUCTION}

The chemostat is a continuous bioreactor used to culture microorganisms with concentration $x$ which consume a substrate $s$ to grow. Mathematical modeling of chemostat has been extensively developed, mainly using ordinary differential equations, and several results have been validated experimentally. See [1],[22] for a review of mathematical results on the theory of chemostat.

Feedback control of bioreactor models has been a focus of intensive research $[1],[6],[9]$; the problem we investigate in this paper deals with the feedback stabilization of an uncertain chemostat model. In this control context, uncertainty must be understood in two basic types:

(1) Internal: Some parts of the model are not precisely known; for example the functions that describe the kinetic reactions or some parameters.

(2) External: The measure of the variables is susceptible to undergo different kinds of noise such as unknown additive or multiplicative disturbances.

We shall present two examples of robust stabilization of an uncertain chemostat: The first one is a water depollution process and the second one is the culture of phytoplankton and the simulation of marine environments.

- We can consider the chemostat as a simple wastewater plant (see for example [21],[24]), where a selected microorganism $x$ consumes a contaminant $s$ and is expected to make decrease its concentration toward an acceptable level given by environmental laws. This goal is considerably complicated by uncertainties of the kinetic model and the output.

- Chemostat has been used currently in marine laboratories (see [2],[3]) to simulate in vitro the growth of unicellular phytoplanktonic algae in marine

Date: 4 August 2005.

1991 Mathematics Subject Classification. 34D23,34H05,93B52.

Key words and phrases. Chemostat, Uncertain models, Feedback control, Monotone systems. 
ecosystems, where the phytoplankton feeds on limiting substrate (e.g. nitrate, iron, silicon, etc.) supplied at a constant rate. As it is easy to see, the standard equilibrium point in a chemostat with a monotone classical growth function as the Michaelis-Menten function corresponds to a low level of substrate: it comes from the fact that the growth function has a large slope at the origin, attaining very fast its maximum. In fact, experimentally, the value of the substrate at the equilibrium is often so small that it is non measurable with the usual apparatus. To reproduce growth conditions with a high level of nutrient in vitro, we wish to be able to maintain a high level of substrate at the equilibrium. At this level, the risk of washout (loss of biomass) is very high if there are uncertainties in the model. Therefore we need a control to stabilize the chemostat and avoid washout.

Feedback stabilization of a chemostat becomes nontrivial if we take into account that the model is inaccurate, thus the real dynamics is ill-known and the output available is corrupted by noise.

Feedback stabilization of nonlinear uncertain systems [4],[15] deals with several approaches: e.g. deterministic, stochastic, adaptive control, etc: in [4] it is supposed that the behavior of the uncertainties can be described by differential equations. Stochastic control (see [15, Ch.4]) supposes that the uncertainties satisfy some statistical properties. In this article we will only suppose that the uncertainties are deterministic and bounded.

An interesting deterministic issue for this stabilization problem has been given in the framework of the game-theoretical control theory (see e.g. [14]). Roughly speaking, it assumes that the real system is not known to us, but there exists a (relatively wide) class of admissible systems, including the real one. This assumption implies that, given a stabilization objective, it must be satisfied for any system too. This idea has been complemented with a worst case approach (see $[7],[8],[17],[19],[20])$ by using the fact that (under some transformation of variables) a system $\Sigma$ (e.g. bioreactor equations) can be studied using monotone dynamical systems theory [23]. Moreover, supposing that the bounds of uncertainties are known a priori, a couple of well known systems $\left(\Sigma^{-}, \Sigma^{+}\right)$verifying the inequality $\Sigma^{-} \leq \Sigma \leq \Sigma^{+}$(in a sense that will be explained later on) is built. Hence, instead of satisfying a feedback stabilization objective for any admissible system, we will need only to satisfy this one for the "bounds" of the admissible systems.

Using these systems $\left(\Sigma^{-}, \Sigma^{+}\right)$, interval observers for uncertain bioreactor models have been developed in [7],[8],[19],[20], allowing a partial estimation for the nonmeasured variables. Moreover, in [20] a feedback stabilization law is built using these interval observers. In almost all those articles (except [19]) it is supposed that the output available is unperturbed and the mortality rate of the biomass(es) is negligible. This last assumption is one key step in reducing many chemostat models to a monotone dynamical systems where strong convergence properties are in evidence.

In this paper, we assume that the output undergoes multiplicative disturbances. Moreover, we drop the assumption of neglecting the mortality rate. Nevertheless, we are able to follow the ideas stated above, arriving to stabilize the uncertain chemostat without using interval observers. 
This paper is organized as follows: in section 2 we recall some facts of the chemostat model and state the assumptions about uncertainty. Section 3 presents the robust regulation problem in detail. The main result and its proof is given in section 4 . The application and simulations are given in section 5 .

\section{Modeling OF AN UnCERTAin CHEMOSTAT}

Let us recall the chemostat equations (see, for example [22, Chapt.1]):

$$
\left\{\begin{array}{l}
\dot{s}=D\left(s_{i n}-s\right)-\alpha x f(s), \\
\dot{x}=x(f(s)-D-m) .
\end{array}\right.
$$

Where $s(t)$ and $x(t)$ are the concentration of the nutrient and the density of the biomass at time $t, s_{i n}>0$ denotes the input concentration of nutrient (in this paper we will suppose that is a constant), $D>0$ is the dilution rate, $\alpha>0$ is a growth yield constant. The function $f: \mathbb{R}_{+} \mapsto \mathbb{R}_{+}$represents the per capita growth rate of nutrient of the biomass and $m>0$ is the mortality rate. In general, the mortality rate is neglected and a "conservation law" for total biomass implies that the weighted sum of microbial concentration and substrate concentration equilibrates. Hence, the substrate equation can be asymptotically eliminated.

Chemostat is called also CSTR (continuous stirred tank reactor) because, as we can deduce from system (1), the rate of the input flow is the same as that the rate of the output flow. We will assume that initial conditions of system (1) are in the box $\left[s_{0}^{-}, s_{0}^{+}\right] \times\left[x_{0}^{-}, x_{0}^{+}\right] \subset \Omega$, where the set $\Omega$ is defined by:

$$
\Omega=\left\{(s, x) \in \mathbb{R}_{+}^{2}: 0<s<s_{\text {in }}, x>0 \text { and } s+\alpha x+s_{\text {in }}<v^{*}\right\}
$$

where $v^{*}$ is a constant related to the volume of the chemostat.

Now, we make the assumptions about uncertainty of chemostat model:

(H1) The function $f$ is unknown but locally Lipschitz and functionally bounded, i.e. there exist a couple of well known maps $f^{-}$and $f^{+}$such that:

- $f^{-}$and $f^{+}$are continuous and have at most one local maximum in $\left(0, s_{i n}\right)$. - $f^{-}$and $f^{+}$satisfy the inequalities (See Fig.1):

$$
f^{-}(s) \leq f(s) \leq f^{+}(s), \quad \text { for any } s \geq 0 \text { and } \quad f^{-}(0)=f^{+}(0)=0 .
$$

(H2) The only output available takes the form:

$$
y(t)=s(t)[1+\Delta(t)]
$$

where the function $\Delta: \mathbb{R}_{+} \mapsto \mathbb{R}$ is bounded and Lebesgue measurable. Moreover, there exist two bounds for $\Delta(t)$ :

$$
-1<\Delta^{-} \leq \Delta(t) \leq \Delta^{+}
$$

Output $y(t)$ is known as a deterministic multiplicative disturbance (see e.g. [18]).

It will be useful to define the lower and upper increasing envelope of the functions $f^{-}$and $f^{+}$respectively, that means a couple of continuous and increasing functions $l$ and $u$, satisfying $l(s) \leq f^{-}(s) \leq f^{+}(s) \leq u(s)$ for any $s \in\left[0, s_{i n}\right]$ (see Fig.2).

Remark 1. (i) Assumption (H1) follows from the fact that the explicit formulation of the function $f$ is based on experimental evidence. Indeed, in several experiments 


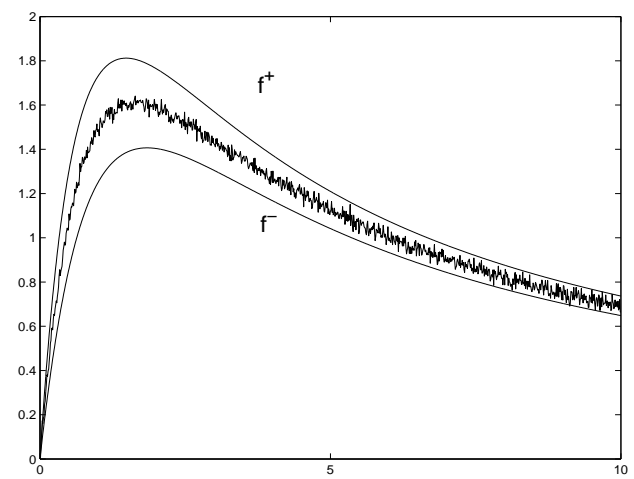

Figure 1. Geometrical interpretation of (H1) x-axis: Substrate; y-axis: $f$ and its upper bounds

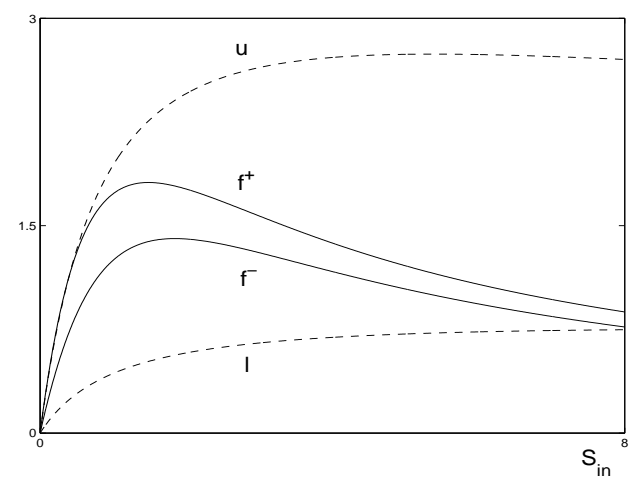

Figure 2. $l$ is a lower envelope of $f^{-}$and $u$ is an upper envelope of $f^{+}$

it has been observed that according to substrate, the Michaelis-Menten function or the Haldane function (see [22]) defined respectively by:

$$
f(s)=\frac{\mu_{m} s}{s+k_{s}} \quad \text { and } \quad f(s)=\frac{\mu_{m} s}{s+k_{s}+\frac{s^{2}}{k_{i}}} \quad \mu_{m}, k_{s}, k_{i}>0
$$

provide a reasonable approximation for the experimental data. $\mu_{m}$ is the maximum specific growth rate of limiting nutrient, $k_{s}$ is the half-saturation constant (also called Monod's constant) and $k_{i}$ is the inhibition constant. Normally, there are uncertainty in the identification of these kinetic parameters (see for example [1], [6, Chapt.3-5],[12][21]).

(ii) Assumption (H2) is very common in the measure of biological variables: the information about the state variables is restricted and the available data are few and biased, the inequality $\Delta^{-}>-1$ follows from the fact that the outputs are always positive. Notice that there is not any statistical assumption for disturbance $\Delta(t)$ because every measurable function between $\left[\Delta^{-}, \Delta^{+}\right]$is considered to be as good as any other one: we need only deterministic bounds on the uncertainties. 
Moreover, (H2) gives a priori bounded estimates for the substrate:

$$
\frac{y(t)}{1+\Delta^{+}} \leq s(t) \leq \frac{y(t)}{1+\Delta^{-}} .
$$

Recall that (H1) and (H2) will be assumed for the remainder of this paper.

\section{MOTIVATION AND FORMULATION OF STABILIZATION PROBLEM}

In several bioprocesses, the goal is to stabilize the substrate in a neighborhood of level $s^{*}$; moreover it is important to produce an estimation -even during the transient of systems- for the unmeasured variable $x$. Nevertheless this task is made difficult by the uncertainties of the model summarized by (H1) $-(\mathbf{H 2})$.

We wish to motivate the abstract work that follows, and display two concrete examples.

- A model of depollution using a chemostat: Water quality management is facing enormous difficulties in conserving standard levels in order to keep it usable for drinking and tourism. In this context, a considerable interest has been focused in toxic contaminants and its stabilization under an acceptable level.

Phenol is a troublesome contaminant in surface water and biological oxidation of phenol has been an important waste treatment process for years. Briefly, this process of depollution consists in a continuous well-stirred tank in which the phenol is pumped into with a concentration $s_{i n}$, higher than an acceptable level of the contaminant in the water defined by $s^{+}$(relatively near to zero) and fixed by environmental authorities. This tank also contains a microorganism $x$, which can resist the adverse effects of organic solvents as phenol and is capable of decontamining the tank because it is able to utilize phenol as limiting substrate.

Notice that, if we choose a fixed dilution $D<f^{+}\left(s^{+}\right)$, the concentration of contaminant will be convergent to an acceptable level. Nevertheless, as the dilution rate is relatively small, the convergence toward the equilibrium point is too slow, furthermore numerical simulations and linear analysis around the critical points suggest that the introduction of a feedback control law increases the speed of convergence.

- Simulation of marine environment using a chemostat: Chemostat has been used to model some aspects of marine environment (see e.g. [2],[3]). In fact, several features of the sea (e.g. temperature and light intensity) can be reproduced in the laboratory. Moreover, controlling chemostat allows reproduction of several fixed levels $s^{*}$ of limiting substrate, and consequently, makes possible to study the growth of unicellular phytoplanktonic algae in a wide range of substrate levels, temperature and/or brightness.

We are interested in stabilize the chemostat to a high level of limiting substrate $s^{*}$ close to $s_{i n}$ to reproduce high levels of nitrate under the sea. Notice that, if we choose a dilution rate $D$ near to $f^{+}\left(s^{*}\right)$ and the function $f^{-}$is a Michaelis-Menten type function satisfying $D>f^{-}\left(s_{i n}\right)$, the critical point $E_{0}=\left(s_{i n}, 0\right)$ becomes a globally stable solution of system (1) taking $f=f^{-}$, forcing the washout of biomass from the chemostat.

The washout of biomass must be avoided in any experiment concerning unicellular phyoplanktonic algae because it will be necessary to start the experiment again 
with a loss of time and material. So, we introduce a feedback control law that stabilizes the output in an interval $\left[s^{-}, s^{+}\right]$containing $s^{*}$.

Motivated by these technical difficulties, we formulate the following problem:

Problem 1 (The robust regulation problem $\mathcal{P}$ ). Given a reference value $s^{*} \in$ $\left(0, s_{i n}\right)$ and considering $D$ as a feedback control variable; find a family of positive feedback control laws $D: \mathbb{R}_{+} \times \Omega \mapsto \mathbb{R}_{+} \backslash\{0\}$ such that the closed-loop system (1) has the following properties:

(a) There exist two bounded intervals, an upper one and a lower one, for the unmeasured variable $x(t)$ and the substrate $s(t)$, that improve the estimation given by Eq.(2). That means a set of well known functions $s^{-}, s^{+}, x^{-}$and $x^{+}: \mathbb{R}_{+} \mapsto \mathbb{R}_{+}$such that:

$x^{-}(t) \leq x(t) \leq x^{+}(t) \quad$ and $\quad s^{-}(t) \leq s(t) \leq s^{+}(t)$ for any $t \geq 0$.

(b) There exists a compact set $K=\left[s^{-}, s^{+}\right] \times\left[x^{-}, x^{+}\right] \in \Omega$ and a finite time $T \geq 0$ such that $(s(t), x(t)) \in K$ for any $t>T$; moreover $s^{*} \in\left(s^{-}, s^{+}\right)$.

There exists some problems related to $(\mathcal{P})$ : in [19], the system $(1)$ is studied under the following assumptions: $m=0,(\mathbf{H 1})$ holds and the only output available is the substrate with additive perturbations i.e. $y(t)=s(t)+\Delta(t)$. Using monotone dynamical systems theory, an interval observer has been built, allowing the estimation of the non-measured variable (the biomass).

In [20], a more general bioreactor is studied under the following assumptions: $m=0$, (H1) holds and the only output available is the substrate i.e. $y(t)=s(t)$. The non-measured variables are estimated with an interval observer and using these estimations, a feedback control law that stabilizes the substrate $s(t)$ in a neighborhood of the reference value $s^{*}$ has been built.

\section{Feedback control laW}

Let us consider the following family of feedback control laws:

$$
D(y(t))=D^{*}+h(y(t))
$$

where $D^{*}$ is a constant satisfying the inequality $f^{-}\left(s^{*}\right)<D^{*}+m<f^{+}\left(s^{*}\right)$ and the function $h: \mathbb{R} \mapsto \mathbb{R}$ satisfies the following assumptions (G):

(G1) $h$ is Lipschitz, decreasing such that:

$$
h\left(s^{*}\right)=0, \quad-D^{*}<h\left(s_{i n}\left[1+\Delta^{+}\right]\right)<h\left(s_{i n}\right)<l\left(s_{i n}\right)-\left(D^{*}+m\right) .
$$

(G2) The bounds $\Delta^{-}$and $\Delta^{+}$are such that the equations:

$$
\begin{aligned}
& D^{*}-u(s)+h\left(s\left[1+\Delta^{+}\right]\right)+m\left\{\frac{D^{*}+h\left(s\left[1+\Delta^{+}\right]\right)}{D^{*}+h\left(s\left[1+\Delta^{-}\right]\right)}\right\}=0, \\
& D^{*}-l(s)+h\left(s\left[1+\Delta^{-}\right]\right)+m\left\{\frac{D^{*}+h\left(s\left[1+\Delta^{-}\right]\right)}{D^{*}+h\left(s\left[1+\Delta^{+}\right]\right)}\right\}=0
\end{aligned}
$$

have one single root $s^{l} \in\left(0, s^{*}\right)$ and $s^{u} \in\left(s^{*}, s_{i n}\right)$ respectively.

(G3) The death rate satisfies the following inequalities:

$$
m<\inf _{r \in\left(0, s_{i n}\right)}\left\{l(r) \frac{h^{\prime}\left(r\left[1+\Delta^{+}\right]\right)}{h^{\prime}\left(r\left[1+\Delta^{-}\right]\right)} \frac{1+\Delta^{+}}{1+\Delta^{-}}-l^{\prime}(r) \frac{D^{*}+h\left(r\left[1+\Delta^{-}\right]\right)}{\left[1+\Delta^{-}\right] h^{\prime}\left(r\left[1+\Delta^{-}\right]\right)}\right\},
$$




$$
m<\inf _{r \in\left(0, s_{i n}\right)}\left\{u(r) \frac{h^{\prime}\left(r\left[1+\Delta^{-}\right]\right)}{h^{\prime}\left(r\left[1+\Delta^{+}\right]\right)} \frac{1+\Delta^{-}}{1+\Delta^{+}}-u^{\prime}(r) \frac{D^{*}+h\left(r\left[1+\Delta^{+}\right]\right)}{\left[1+\Delta^{+}\right] h^{\prime}\left(r\left[1+\Delta^{+}\right]\right)}\right\} .
$$

(G4) The death rate satisfies the following inequality:

$$
m<\min \left\{s_{i n} \frac{D^{*}+h(0)}{v^{*}+s_{i n}},\left(\frac{s_{i n}-s^{u}}{s_{i n}-s^{l}}\right)\left[D^{*}+h\left(s^{u}\left[1+\Delta^{+}\right]\right)\right]\right\} .
$$

Remark 2. Notice that assumption (G1) can always be satisfied with reasonable choices of $h$. Moreover, Eq.(2) implies that the output $y(t)$ is bounded by the interval $\left[s_{i n}\left(1+\Delta^{-}\right), s_{i n}\left(1+\Delta^{+}\right)\right]$. Hence, assumption (G1) implies that $D(y(t))$ is defined on a bounded interval, we will be able to fulfill the physical constraints:

$$
0<\underbrace{D^{*}+h\left(s_{i n}\left[1+\Delta^{+}\right]\right)}_{D_{\min }}<D(y(t))<\underbrace{D^{*}+h\left(s_{i n}\left[1+\Delta^{-}\right]\right)}_{D_{\max }} \quad \text { for any } t \geq 0 .
$$

Assumption (G2) can be satisfied if $\left|\Delta^{-}\right|$and $\left|\Delta^{+}\right|$are relatively small. Indeed, notice that if $\Delta^{-}=\Delta^{+}=0$, the equations stated in (G2) becomes:

$$
D^{*}-u(s)+h(s)+m=0 \text { and } \quad D^{*}-l(s)+h(s)-m=0,
$$

by using (G1), it is straightforward to prove that these equations have only one single root $\tilde{s}^{l} \in\left(0, s^{*}\right)$ and $\tilde{s}^{u} \in\left(s^{*}, s_{i n}\right)$. Finally, using implicit function theorem we can prove the existence of a bound $\Delta_{0}>0$ such that the inequality $\max \left\{\left|\Delta^{-}\right|, \Delta^{+}\right\}<\Delta_{0}$ implies (G2).

Remark 3. Assumptions (G3)-(G4) gives an upper bound for the mortality rate. Notice that mortality rate $m$ cannot exceed some threshold, otherwise the solution $\left(s_{i n}, 0\right)$ of system (1) could become globally attractive. Moreover, notice that when $m$ is relatively small with respect to $D_{\text {min }}$, assumptions $(\mathbf{G} 3)-(\mathbf{G} 4)$ can be satisfied with reasonable choices of $h$.

4.1. Main result. In this section, we give sufficient conditions to solve the problem $(\mathcal{P})$ summarized in Theorem 1. The key idea of the proof is to transform the closedloop system (1) into a system that can be compared with cooperative systems i.e. a system such that the off diagonal entries of the Jacobian matrix are nonnegative. Planar cooperative systems theory (see Appendix) will be the main tool employed.

Theorem 1. The problem $(\mathcal{P})$ is solvable by a family of output feedback control defined by (3) satisfying (G1)-(G4).

Proof. We will verify the properties (a) and (b) separately.

Step1: Replacing $D$ by $D(y(t))$, system (1) becomes:

$$
\left\{\begin{array}{l}
\dot{s}=\left\{D^{*}+h(s[1+\Delta(t)])\right\}\left(s_{i n}-s\right)-\alpha x f(s), \\
\dot{x}=x\left[f(s)-D^{*}-h(s[1+\Delta(t)])-m\right], \\
(s(0), x(0)) \in\left[s_{0}^{-}, s_{0}^{+}\right] \times\left[x_{0}^{-}, x_{0}^{+}\right] \subset \Omega .
\end{array}\right.
$$

Notice that, after (H1)-(H2), system (4) satisfies Carathéodory conditions (see e.g. [5, Th 2.1.1]), that guarantees existence and uniqueness of solutions. Moreover, it is straightforward to verify that system (4) is positively invariant in $\Omega$.

Let $(s, x)$ be the solution of system (4). Using a standard argument, we build the function $v: \Omega \mapsto \mathbb{R}$ defined by:

$$
v=s+\alpha x-s_{i n} .
$$


Clearly, it follows that $(x, v)$ is a solution of the system:

$$
\left\{\begin{array}{l}
\dot{x}=x\left[f\left(v+s_{i n}-\alpha x\right)-\left\{D^{*}+h\left(\left[v+s_{i n}-\alpha x\right][1+\Delta(t)]\right\}\right)-m\right], \\
\dot{v}=-\left\{D^{*}+h\left(\left[v+s_{i n}-\alpha x\right][1+\Delta(t)]\right)\right\} v-\alpha m x, \\
x(0)>0, \quad-s_{\text {in }}<v(0)<v^{*} .
\end{array}\right.
$$

We make the time transformation

$$
r=\int_{0}^{t}\left\{D^{*}+h(s(\tau)[1+\Delta(\tau)])\right\} d \tau
$$

Notice that we have build a function $\mathbb{R}_{+} \mapsto \mathbb{R}_{+}$defined by $t \rightarrow r(t)$, Using Remark 2 it can be proved that is invertible. We see at once that $\frac{d x}{d r}=\dot{x}\left\{D^{*}+\right.$ $h(s[1+\Delta(t)])\}^{-1}$ and $\frac{d v}{d r}=\dot{v}\left\{D^{*}+h(s[1+\Delta(t)])\right\}^{-1}$. As this function is injective, we can define also $t(r)$. Hence, the solutions of system (6) can be written as:

$$
\left\{\begin{array}{l}
\frac{d x}{d r}=x\left[\frac{f\left(v+s_{i n}-\alpha x\right)-m}{D^{*}+h\left(\left[v+s_{i n}-\alpha x\right][1+\Delta(r)]\right)}-1\right]=F(r, x, v), \\
\frac{d v}{d r}=-v-\frac{\alpha m x}{D^{*}+h\left(\left[v+s_{i n}-\alpha x\right][1+\Delta(r)]\right)}=G(r, x, v) .
\end{array}\right.
$$

Let us define the set:

$$
\Omega_{1}=\left\{(x, v) \in \mathbb{R}^{2}: x<\alpha^{-1}\left[v+s_{i n}\right], v \in\left(-s_{i n}, v^{*}\right)\right\} .
$$

System (7) is positively invariant in $\Omega_{1}$, now we build the following comparison system in $\Omega_{1}$ :

$$
\left\{\begin{array}{l}
\frac{d \phi}{d r}=\phi\left[\frac{u\left(z+s_{i n}-\alpha \phi\right)}{D^{+}\left(z+s_{i n}-\alpha \phi\right)}-\frac{m}{D^{-}\left(z+s_{i n}-\alpha \phi\right)}-1\right]=F^{+}(\phi, z), \\
\frac{d z}{d r}=-z \quad=G^{+}(\phi, z), \\
x(0) \leq \phi_{0} \quad \text { and } \quad v(0) \leq z_{0} \quad\left(\phi_{0}, z_{0}\right) \in \operatorname{int} \Omega_{1} .
\end{array}\right.
$$

Where the functions $D^{+}, D^{-}: \mathbb{R}_{+} \mapsto \mathbb{R}_{+}$are defined as follow:

$$
D^{+}(s)=D^{*}+h\left(s\left[1+\Delta^{+}\right]\right) \quad \text { and } \quad D^{-}(s)=D^{*}+h\left(s\left[1+\Delta^{-}\right]\right) \quad \text { for any } s \geq 0 .
$$

System (8) is positively invariant in $\Omega_{1}$. Indeed, notice that if there exists a finite time $r_{0}>0$ such that the function $L(r)=z(r)+s_{i n}-\alpha \phi(r)$-where $(\phi, z)$ are solution of the system- verify $L\left(r_{0}\right)=0$, it follows that $\left.\frac{d L}{d r}\right|_{r=r_{0}}>0$ and the invariance is verified. Moreover, by assumption (G3) it follows that system (8) is cooperative. Finally, by (H1)-(H2) and (G1) the inequalities:

$$
F(r, x, v) \leq F^{+}(x, v) \text { and } G(r, x, v) \leq G^{+}(x, v)
$$

follow for any $(r, x, v) \in \mathbb{R}_{+} \times \Omega_{1}$.

Applying comparison theorem for cooperative systems (see Prop.1 in the Appendix) to the systems (7) and (8), we see that:

$$
x(r) \leq \phi(r) \quad \text { and } \quad v(r) \leq z(r) \quad \text { for any } r \geq 0 .
$$

Let $(\phi(r), z(r))$ be the solution of system (8). Now, we use this function $\phi(r)$ to build the following comparison system in $\Omega_{1}$ : 


$$
\left\{\begin{array}{l}
\frac{d \eta}{d r}=\eta\left[\frac{l\left(\chi+s_{i n}-\alpha \eta\right)}{D^{-}\left(\chi+s_{i n}-\alpha \eta\right)}-\frac{m}{D^{+}\left(\chi+s_{i n}-\alpha \eta\right)}-1\right]=F^{-}(\eta, \chi), \\
\frac{d \chi}{d r}=-\chi-\frac{\alpha m \phi(r)}{D^{+}\left(\chi+s_{i n}-\alpha \eta\right)} \\
0<\eta_{0} \leq x(0) \text { and } \chi_{0} \leq v(0) \text { and }\left(\eta_{0}, \chi_{0}\right) \in \operatorname{int} \Omega_{1} .
\end{array}\right.
$$

Notice that by (G4) we have that system (10) is positively invariant in $\Omega_{1}$. Moreover, it follows from assumption (G3) that system (10) is cooperative. Finally, assumptions (H1) $-(\mathbf{H 2}),(\mathbf{G 1})$ and Eq.(9) imply that:

$$
F^{-}(x, v) \leq F(r, x, v) \quad \text { and } \quad G^{-}(r, x, v) \leq G(r, x, v)
$$

follow for any $(r, x, v) \in \mathbb{R}_{+} \times \Omega_{1}$.

Applying again Prop.1 to systems (7) and (10), we see that:

$$
\eta(r) \leq x(r) \quad \text { and } \quad \chi(r) \leq v(r) \quad \text { for any } r \geq 0 .
$$

Using Eqs.(9) and (11), the functional bounds for the biomass are:

$$
x^{-}(r)=\eta(r) \leq x(r) \leq \phi(r)=x^{+}(r) .
$$

Moreover, using Eq.(5), the estimation for the substrate given by Eq.(2) can be improved by $s^{-}(r) \leq s(r) \leq s^{+}(r)$, where $s^{-}(r)$ and $s^{+}(r)$ are defined by:

$s^{-}(r)=\max \left\{s_{i n}-\alpha \phi(r)+z(r), y^{-}(r)\right\}, \quad s^{+}(r)=\min \left\{s_{i n}-\alpha \eta(r)+\chi(r), y^{+}(r)\right\}$.

Indeed, to obtain the bounds on $s(t)$, we take the minimum of the bounds given by the output and of those given by the comparison systems and property (a) is verified.

Step 2: In order to verify property (b), notice that assumptions (G2) and (G4) imply that the critical points of system (8) are:

$$
E_{1}^{+}=\left(\alpha^{-1}\left[s_{i n}-s^{l}\right], 0\right) \text { and } E_{2}^{+}=(0,0) .
$$

A linearization procedure combined with assumptions (G2)-(G4) shows that $E_{1}^{+}$and $E_{2}^{+}$are respectively locally stable and unstable.

It can be proved that the critical point $E_{2}^{+}$cannot be an $\omega$-limit set for any initial condition $\left(\phi_{0}, z_{0}\right)$. We will sketch this proof:

- It is straightforward to verify that the stable manifold of the critical point $E_{2}^{+}$is defined by the set:

$$
W^{s}\left(E_{2}^{+}\right)=\left\{(\phi, z) \in \Omega_{1}: \phi=0\right\} .
$$

- We build the functional $P: \Omega_{1} \mapsto \mathbb{R}$ defined by $P(\phi, z)=\phi$. Clearly $P=0$ in $W^{s}\left(E_{2}^{+}\right)$and $P>0$ in $\Omega_{1} \backslash W^{s}\left(E_{2}^{+}\right)$.

- It follows from system (8) that $\dot{P}=\Psi(\phi, z) P$ where $\Psi: \Omega_{1} \mapsto \mathbb{R}$ is the continuous function:

$$
\Psi(\phi, z)=\frac{u\left(z+s_{i n}-\alpha \phi\right)}{D^{*}+h\left(\left[z+s_{i n}-\alpha \phi\right]\left[1+\Delta^{+}\right]\right)}-\frac{m}{D^{*}+h\left(\left[z+s_{\text {in }}-\alpha \phi\right]\left[1+\Delta^{-}\right]\right)}-1 .
$$

- It follows from (G2) that $\Psi\left(E_{2}^{+}\right)>0$. This imply that $P$ is an average Lyapunov function (see e.g. [10],[11]) and using Th.12.2.2 from [11] it follows that $E_{2}^{+}$cannot be attained from int $\Omega_{1}$.

Since the solutions of system (10) are bounded, applying Prop.2, it follows that:

$$
\lim _{r \rightarrow+\infty}(\phi(r), z(r))=E_{1}^{+} .
$$


Hence system (10) is asymptotically autonomous (see for example [25],[26] and the references given there) with limit system:

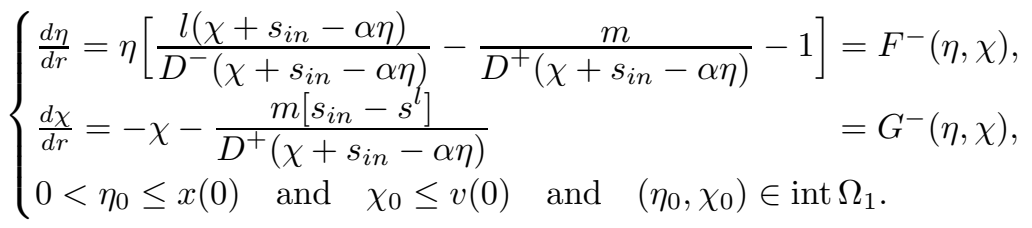

Assumptions (G3)-(G4) imply that the system (12) is positively invariant and cooperative in $\Omega_{1}$. Moreover, its critical points are:

$$
E_{1}^{-}=\left(\alpha^{-1}\left[s_{i n}-s^{u}+\xi_{0}\left(\Delta^{+}\right)\right], \xi_{0}\left(\Delta^{+}\right)\right) \quad \text { and } \quad E_{2}^{-}=(0, \bar{\chi})
$$

where $\xi_{0}\left(\Delta^{+}\right)=\frac{-m\left(s_{i n}-s^{l}\right)}{D^{*}+h\left(s^{u}\left[1+\Delta^{+}\right]\right)}$and $\bar{\chi} \in\left(s^{u}-s_{i n}, 0\right)$ is the unique root of the function $\left(-s_{i n}, 0\right) \mapsto \mathbb{R}$ defined by

$$
r \mapsto-r-\frac{m\left(s_{i n}-s^{l}\right)}{D^{*}+h\left(\left[r+s_{i n}\right]\left[1+\Delta^{+}\right]\right)} .
$$

By assumptions (G2)-(G4) combined with a linearization procedure, it follows that $E_{1}^{-}$and $E_{2}^{-}$are respectively locally stable and unstable. Moreover, following the lines of the proof given for the point $E_{2}^{+}$, we can prove that the critical point $E_{2}^{-}$cannot be an $\omega$-limit set for any initial condition $\left(\eta_{0}, \chi_{0}\right)$.

Since the solutions of system (8) are bounded and Th.1 implies that $E_{1}^{-}$is a global attractor of system (12), it follows by Poincaré-Bendixson trichotomy for asymptotically autonomous systems (see for example [25, Th.1.6],[26, Th.1.5]) that:

$$
\lim _{r \rightarrow+\infty}(\eta(r), u(r))=E_{1}^{-} .
$$

Combining these estimates with Eq.(5) gives that property (b) is verified with:

$$
K=\left[s^{l}, s^{u}\right] \times\left[\alpha^{-1}\left(s_{i n}-s^{u}+\xi_{0}\left(\Delta^{+}\right)\right), \alpha^{-1}\left(s_{i n}-s^{l}\right)\right] .
$$

4.2. Some extensions. This subsection deals with some improvements of the main result. Notice that the area of the set $K$ is $A(K)=\alpha^{-1}\left(s^{u}-s^{l}\right)\left[\left(s^{u}-s^{l}\right)-\xi_{0}\left(\Delta^{+}\right)\right]$ and using (G2) we can realize that $A(K)$ is strongly determinate by the feedback control law chosen. So, motivated by practical applications, it will be desirable to reduce the area of $K$ by choosing an adequate feedback control law. In this sense, we have the following result:

Corollary 1. Given $\varepsilon>0$, there exists an appropriate function $h$ such that:

$$
\left|\frac{s^{l}}{s^{u}}-\frac{1+\Delta^{-}}{1+\Delta^{+}}\right|<\varepsilon .
$$

Proof. We can choose a neighborhood $V$ of $s^{*}$ such that $\left(s^{-}, s^{+}\right) \subset V$. Hence, using (G1)-(G2) and mean value theorem we obtain:

$$
\left|\frac{s^{u}}{s^{l}}-\frac{1+\Delta^{+}}{1+\Delta^{-}}\right|=\frac{\left|u\left(s^{l}\right)-l\left(s^{u}\right)\right|+m\left[\frac{D^{+}\left(s^{l}\right)}{D^{-}\left(s^{l}\right)}+\frac{D^{-}\left(s^{u}\right)}{D^{+}\left(s^{u}\right)}\right]}{s^{u}\left|h^{\prime}(\rho)\right|\left[1+\Delta^{+}\right]} \leq \frac{C}{\left|h^{\prime}(\rho)\right|}
$$


for some $\rho \in\left(s^{l}, s^{u}\right)$ and $C>0$ is a constant defined by

$$
C=\left(s^{*}\left[1+\Delta^{+}\right]\right)^{-1}\left[\max _{r \in\left[0, s_{i n}\right]}|u(r)-l(r)|+2 m \frac{D_{\max }}{D_{\min }}\right] .
$$

Now, choosing a control law such that $\left|h^{\prime}(u)\right|>C \varepsilon^{-1}$ for any $u \in V$ completes the proof.

Moreover, we can build a decreasing sequence of sets $\left\{K_{j}\right\}_{j}\left(K_{0}=K\right)$ of sets verifying $A\left(K_{j+1}\right) \subset A\left(K_{j}\right)$ for any integer $j \geq 0$. This is the content of the following result:

Corollary 2. There exist two sequences $\left\{\eta_{j}\right\}_{j},\left\{\phi_{j}\right\}_{j}$ of functions in $C\left(\mathbb{R}_{+}, \mathbb{R}_{+}\right)$ satisfying the following properties:

(i) The first one is nonnegative and the second one is upperly bounded by $x_{0}^{+}$, and they satisfy the inequalities:

$0 \leq \eta_{j-1}(r) \leq \eta_{j}(r) \leq \ldots \leq x(r) \leq \ldots \leq \phi_{j}(r) \leq \phi_{j-1}(r) \leq \alpha^{-1}\left(v^{*}+s_{i n}\right)$

for any integer $j \geq 1$ and $r \geq 0$.

(ii) The functions have the asymptotic behavior for any integer $j \geq 0$ :

$\lim _{r \rightarrow+\infty} \phi_{j}(r)=\frac{s_{i n}-s^{l}+\xi_{j}\left(\Delta^{-}\right)}{\alpha}$ and $\lim _{r \rightarrow+\infty} \eta_{j}(r)=\frac{s_{i n}-s^{u}+\xi_{j}\left(\Delta^{+}\right)}{\alpha}$

where the sequences $\xi_{j}\left(\Delta^{-}\right)$and $\xi_{j}\left(\Delta^{+}\right)$are recursively defined by:

$$
\begin{aligned}
& \xi_{j}\left(\Delta^{-}\right)= \begin{cases}0 & \text { if } j=0, \\
-\frac{m\left[s_{i n}-s^{u}+\xi_{j-1}\left(\Delta^{+}\right)\right]}{D^{*}+h\left(s^{l}\left[1+\Delta^{+}\right]\right)} & \text {if } j \in\{1,2, \ldots\} .\end{cases} \\
& \xi_{j}\left(\Delta^{+}\right)=-\frac{m\left[s_{i n}-s^{l}+\xi_{j}\left(\Delta^{-}\right)\right]}{D^{*}+h\left(s^{u}\left[1+\Delta^{-}\right]\right)} \quad j \in\{0,1, \ldots\} .
\end{aligned}
$$

(iii) These sequences are uniformly convergent in $B C([0, \infty), \mathbb{R})$ (the Banach space of bounded continuous functions on $\mathbb{R}_{+}$taking values in $\mathbb{R}$ ) to the functions $\eta_{\infty}$ and $\phi_{\infty}$ respectively.

Proof. (i) For any integer $j \geq 0$, let us build the systems:

$$
\begin{aligned}
& \Sigma_{j}^{+}=\left\{\begin{array}{l}
\frac{d \phi}{d r}=\phi\left[\frac{u\left(z+s_{i n}-\alpha \phi\right)}{D^{+}\left(z+s_{i n}-\alpha \phi\right)}-\frac{m}{D^{-}\left(z+s_{i n}-\alpha \phi\right)}-1\right]=F_{j}^{+}(\phi, z), \\
\frac{d z}{d r}=-z-\frac{\alpha m \gamma_{j}(r)}{D^{-}\left(z+s_{i n}-\alpha \phi\right)} \\
x(0) \leq \phi_{0} \quad \text { and } v(0) \leq z_{0}
\end{array} \quad \text { and } \quad\left(\phi(0), z_{0}\right) \in \operatorname{int} \Omega_{1} .\right.
\end{aligned}
$$

where the functions $\gamma_{j}, \lambda_{j}: \mathbb{R}_{+} \mapsto \mathbb{R}_{+}$verify:

$$
0 \leq \gamma_{j}(r) \leq x(r) \leq \lambda_{j}(r) \leq \alpha^{-1}\left(v^{+}+s_{\text {in }}\right) .
$$

Notice that these systems are cooperative in $\Omega_{1}$. Moreover, given these bounds for $\gamma_{j}(r)$ and $\lambda_{j}(r)$, assumption (G4) implies that systems $\Sigma_{j}^{-}$and $\Sigma_{j}^{+}$are positively invariant in $\Omega_{1}$. 
Let $\left(\phi_{j}(r), z_{j}(r)\right)$ and $\left(\eta_{j}(r), \chi_{j}(r)\right)$ be the solutions of systems $\Sigma_{j}^{+}$and $\Sigma_{j}^{-}$ respectively. Now, we define recursively a particular couple of sequences $\left\{\gamma_{j}\right\}_{j}$ and $\left\{\lambda_{j}\right\}_{j}$ as follows:

$$
\gamma_{j}(r)=\left\{\begin{array}{ll}
0 & \text { if } j=0 \\
\eta_{j-1}(r) & \text { if } j \in\{1,2, \ldots\}
\end{array} \quad \text { and } \quad \lambda_{j}(r)=\phi_{j}(r) \quad j \in\{0,1, \ldots\} .\right.
$$

Now, we will verify (13) and (14) using mathematical induction. Indeed, when $j=0$, systems $\Sigma_{0}^{+}$and $\Sigma_{0}^{-}$are equivalent to (8) and (10) respectively and using Th.1 the inequalities (13) and the limits given by Eq.(14) are verified for $j=0$.

Now, we assume that inequalities (13) and the limits given by Eq.(14) are verified for any integer $j \in\{0, \ldots, k-1\}$, we will prove that they are verified also for $j=k$.

Hence, it follows that the inequalities:

$$
F_{k}^{+}(r, \phi, z) \leq F_{k-1}^{+}(r, \phi, z) \quad \text { and } \quad G_{k}^{+}(r, \phi, z) \leq G_{k-1}^{+}(r, \phi, z)
$$

hold for any $(r, \phi, z) \in \mathbb{R}_{+} \times \Omega_{1}$.

Using this inequality and applying Prop. 1 to systems $(7), \Sigma_{k-1}^{+}$and $\Sigma_{k}^{+}$, it follows that $x(r) \leq \phi_{k}(r) \leq \phi_{k-1}(r)$.

This last statement implies that:

$$
F_{k-1}^{-}(r, \eta, \chi) \leq F_{k}^{-}(r, \eta, \chi) \quad \text { and } \quad G_{k-1}^{-}(r, \eta, \chi) \leq G_{k}^{-}(r, \eta, \chi)
$$

hold for any $(r, \eta, \chi) \in \mathbb{R}_{+} \times \Omega_{1}$.

Now, applying again Prop. 1 to systems $(7), \Sigma_{k-1}^{-}$and $\Sigma_{k}^{-}$, it follows that $\eta_{k-1}(r) \leq$ $\eta_{k}(r) \leq x(r)$ and the inequalities (13) are verified for $j=k$.

(ii) Notice that systems $\Sigma_{j}^{+}$and $\Sigma_{j}^{-}$are asymptotically autonomous with limits:

$$
\begin{aligned}
& \tilde{\Sigma}_{j}^{+}=\left\{\begin{array}{l}
\frac{d \phi}{d r}=\phi\left[\frac{u\left(z+s_{i n}-\alpha \phi\right)}{D^{+}\left(z+s_{i n}-\alpha \phi\right)}-\frac{m}{D^{-}\left(z+s_{i n}-\alpha \phi\right)}-1\right]=\widetilde{F}_{j}^{+}(\phi, z), \\
\frac{d z}{d r}=-z-\frac{m\left[s_{i n}-s^{u}-\xi_{j-1}\left(\Delta^{+}\right)\right]}{D^{-}\left(z+s_{i n}-\alpha \phi\right)} \\
x(0) \leq \phi_{0} \quad \text { and } v(0) \leq z_{0} \quad \text { and } \quad\left(\phi_{0}, z_{0}\right) \in \operatorname{int} \Omega_{1} .
\end{array}\right. \\
& \widetilde{\Sigma}_{j}^{-}=\left\{\begin{array}{l}
\frac{d \eta}{d r}=\eta\left[\frac{l\left(\chi+s_{i n}-\alpha \eta\right)}{D^{-}\left(\chi+s_{i n}-\alpha \eta\right)}-\frac{m}{D^{+}\left(\chi+s_{i n}-\alpha\right)}-1\right]=\widetilde{F}_{j}^{-}(\eta, \chi), \\
\frac{d \chi}{d r}=-\chi-\frac{m\left[s_{i n}-s^{l}-\xi_{j}\left(\Delta^{-}\right)\right]}{D^{+}\left(\chi+s_{i n}-\alpha \eta\right)} \\
x(0) \geq \eta_{0} \quad \text { and } \quad v(0) \geq \chi_{0} \quad \text { and } \quad\left(\eta_{0}, \chi_{0}\right) \in \operatorname{int} \Omega_{1} .
\end{array}\right.
\end{aligned}
$$

Using again the results given by the Poincaré-Bendixson trichotomy and following the lines of step 2 in the proof of Theorem 1 we can prove that the solutions of systems $\Sigma_{j}^{+}$and $\Sigma_{j}^{-}$are respectively convergent to the points $E_{j}^{+}$and $E_{j}^{-}$defined as follows:

$E_{j}^{+}=\left(\frac{s_{i n}-s^{l}+\xi_{j}\left(\Delta^{-}\right)}{\alpha}, \xi_{j}\left(\Delta^{-}\right)\right) \quad$ and $\quad E_{j}^{-}=\left(\frac{s_{i n}-s^{u}+\xi_{j}\left(\Delta^{+}\right)}{\alpha}, \xi_{j}\left(\Delta^{+}\right)\right)$.

(iii) Now, we will prove that the sequences $\left\{\eta_{j}\right\}_{j}$ and $\left\{\phi_{j}\right\}_{j}$ are uniformly convergent to a couple of functions $\eta_{\infty}$ and $\phi_{\infty}$ verifying the inequality (13). We only prove the result for the sequence $\left\{\eta_{j}\right\}_{j}$, the other case can be proved analogously.

Firstly, given any $T>0$, we study the properties of the sequence $\left\{\eta_{j}\right\}_{j}$ in the interval $[0, T]$. Notice that, by using the systems $\Sigma_{j}^{-}$and $\Sigma_{j}^{+}$we can deduce that the Lipschitz constant for this sequence is given by a number $L \leq$ $\alpha^{-1} v^{*}\left(l\left(s_{i n}\right)+m\right) / D_{\min }$ and it is straightforward to verify that the sequence $\left\{\eta_{j}\right\}_{j}$ is an equicontinuous and uniformly bounded set. By using Arzelà-Ascoli theorem, 
we have the existence of a subsequence $\eta_{j_{k}}$ uniformly convergent to a continuous function $\eta \in C([0, T], \mathbb{R})$.

We can suppose that this subsequence verifies $\eta_{j_{k}}(r) \leq \eta_{j_{k+1}}(r)$ for any $r \in[0, T]$. Without loss of generality, we also can suppose that there exist an infinite number of index $j_{k}$ verifying:

$$
\eta_{j_{k}}(r) \leq \eta_{j}(r) \leq \eta_{j_{k+1}}(r) .
$$

Letting $k \rightarrow+\infty$, Eq.(15) implies that $\eta_{j}$ is pointwise convergent to $\eta$ in $[0, T]$. Now, Dini's theorem implies uniform convergence and consequently, $\eta_{j}$ is a Cauchy sequence in $C([0, T], \mathbb{R})$. So, given any $\varepsilon>0$, there exists a number $J_{1}(\varepsilon)>0$ such that:

$$
\left|\eta_{j}(r)-\eta_{j+l}(r)\right|<\varepsilon \quad \text { for any } j>J_{1} \text { and } r \in[0, T] .
$$

Secondly, let $\left\{x_{j}^{-}\right\}_{j}$ be a sequence defined by:

$$
x_{j}^{-}=\alpha^{-1}\left[s_{i n}-s^{l}+\xi_{j}\left(\Delta^{+}\right)\right],
$$

it is straightforward to verify that $\left\{x_{j}^{-}\right\}_{j}$ is convergent to a number $x_{\infty}^{-}$. Hence, given $\varepsilon>0$ there exists $J_{2}(\varepsilon)>0$ such that $\left|x_{j}^{-}-x_{\infty}^{-}\right|<\varepsilon / 4$ for any $j>J_{2}$.

Thirdly, Eqs.(14) imply that given $\varepsilon>0$, there exists a number $T_{J_{2}}(\varepsilon)$ such that $\left|\eta_{j}(r)-x_{j}^{-}\right|<\varepsilon / 4$ for any $r>T_{J_{2}}$.

Now, let $J=\max \left\{J_{1}, J_{2}\right\}$, hence we have that:

$$
\left|\eta_{j}(r)-\eta_{j+l}(r)\right|<\varepsilon \quad \text { for any } j>J \text { and } r>T_{J} .
$$

Finally, putting $T=T_{J}$ in Eq.(16) and combining with Eq.(17) it follows that

$$
\left|\eta_{j}(r)-\eta_{j+l}(r)\right|<\varepsilon \text { for any } j>J \text { and } r \geq 0
$$

and we conclude that $\eta_{j}(r)$ is a Cauchy sequence in $B C([0, \infty), \mathbb{R})$ and the lemma follows.

Remark 4. Corollaries 1 and 2 improve our main result in several ways:

(i) Corollary 1 means that if $\Delta^{+} \approx \Delta^{-} \approx 0$ (small noise in the output), then we are able to stabilize $s$ nearly exactly around $s^{*}$ and $A(K)$ is almost zero.

(ii) The area of the set $K$ is reduced by Corollary 1 , by choosing a feedback control law that minimize $s^{u}-s^{l}$. Moreover, using Corollary 2, we can build a decreasing sequence of sets $K_{j+1} \subset K_{j}\left(K_{0}=K\right)$ verifying $A\left(K_{j+1}\right) \leq A\left(K_{j}\right)$.

(iii) Corollary 2 improves our estimation of the functional bounds for the biomass $x(r)$. Indeed, notice that $\eta_{0}$ and $\phi_{0}$ of the sequence defined in (13) are the functional bounds for the biomass given by Th.1 and as we can see, the two sequences of functions $\left\{\eta_{j}\right\},\left\{\phi_{j}\right\}$ improve the initial estimation.

Moreover, uniform convergence of the sequence, give us the best estimation for the functional bounds for the biomass $x(r)$.

Remark 5. The convergence velocity towards the equilibrium point is increased by the feedback law. Although numerical solutions make it clear (see next section), it is rather difficult to prove formally the fact concerning the nonlinear system. The classical comparison tool consists in linearizing around the equilibria, and comparing the eigenvalues of the system with and without control. Notice that for our system (and taking for simplicity $m=0, \Delta(t)=0$ and $f^{-}=f^{+}=f$ ), we obtain that the two eigenvalues are: 
-Without control: $-1,-\left(s_{i n}-s^{*}\right)\left[f^{\prime}\left(s^{*}\right)\right]$,

-With control $:-1,-\left(s_{i n}-s^{*}\right)\left[h^{\prime}\left(s^{*}\right)+f^{\prime}\left(s^{*}\right)\right]$.

Therefore it can be seen that our feedback control law can (locally) increases the convergence given an eigenvalue as negative as wanted by taking $h^{\prime}(0)$ large.

Remark 6. If the assumption (H1) is a consequence of the the uncertainty of the kinetic parameters, that means, there exists closed intervals $I_{i}(i=1,2,3)$ such that $\mu_{m} \in I_{1}, k_{s} \in I_{2}$ and $k_{i} \in I_{3}$ (see Section 2 and Remark 1), the properties of the systems $\Sigma_{j}^{-}$and $\Sigma_{j}^{+}$make possible to enhance our estimation of the parameters by combining our methods with some adaptive control techniques in the sense that the "static bounds" $f^{-}(s)$ and $f^{+}(s)$ could become "dynamic bounds" $f^{-}(r, s)$ (increasing with respect to $r$ ) and $f^{+}(r, s)$ (decreasing with respect to $r$ ) verifying:

$$
f^{-}(s) \leq f^{-}(r, s) \leq f(s) \leq f^{+}(r, s) \leq f^{+}(s) \text { for any } r, s \geq 0 .
$$

Indeed, using the fact that systems $\Sigma_{j}^{-}$and $\Sigma_{j}^{+}$are cooperative, we can carry out known algorithms (see e.g. [13] and references given there), to obtain new dynamic intervals $\widetilde{I}_{i}(r) \subset I_{i}(i=1,2,3)$ where $\left|\widetilde{I}_{i}(r)\right|$ is decreasing with respect to $r$.

\section{Examples: Depollution of Water and Simulation of marine ENVIRONMENTS}

Let us come back to the problems of stabilization stated before (see Introduction and section 3$)$.

5.1. A model of depollution of phenol in the water. We suppose that $s$ is the phenol and the biomass $x$ is Pseudomonas putida [21], the objective is to stabilize the concentration of phenol below the level $s^{+}$after a finite time $T$. Then, the contaminant concentration becomes upperly bounded:

$$
s(t)<s^{+}<s_{\text {in }} \text { for any } t>T \text {. }
$$

In the following table we present some maximal concentrations allowed by the U.S. environmental protection agency (EPA) and the Canadian evaluation of toxic residues service (SERT) ${ }^{1}$.

\begin{tabular}{|c|l|c|}
\hline$s^{+}(\mathrm{mg} / L)$ & Prevention goals & Reference \\
\hline 0.30 & Avoid pollution (water and organisms) & EPA, 1980 \\
\hline 0.49 & Avoid toxicity (aquatic life) & SERT, 1990 \\
\hline 0.02 & Avoid chronic effects (aquatic life) & SERT, 1990 \\
\hline
\end{tabular}

This model is affected by several uncertainties: the data presented by Sokol and Howell [21], show that the function $f$ that describes the growth of $x(t)$ is of type:

$$
f(s)=\frac{k_{1}(s) s}{k_{2}+s^{2}}
$$

where $k_{1}: \mathbb{R}_{+} \mapsto \mathbb{R}_{+}$is an increasing and bounded function. Despite the evidence for variability of the coefficient $k_{1}$, its precise functional form is unknown. $k_{2}$ is a positive (uncertain) parameter. We summarize the experimental data presented in [21] in the following table (Liters are denoted by $L$ and milligrams by $\mathrm{mg}$ ):

\footnotetext{
${ }^{1}$ See http://www.menv.gouv.qc.ca/eau
} 


\begin{tabular}{|c|l|c|}
\hline Parameter / function & Uncertainty & Units \\
\hline$k_{1}(s)$ & $k_{1}(s) \in[9.43,22.5]$ & Day $^{-1}$ \\
\hline$k_{2}$ & $k_{2} \in[2.82,3.09]$ & $\mathrm{mg} / L$ \\
\hline
\end{tabular}

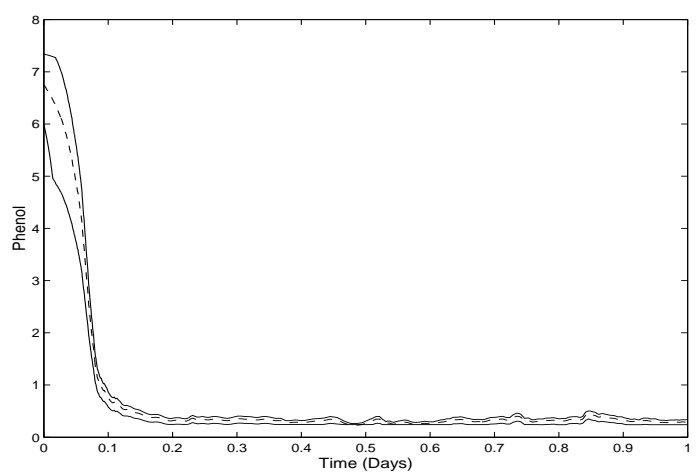

Figure 3. Concentration of phenol (dashed line) and its bounds.

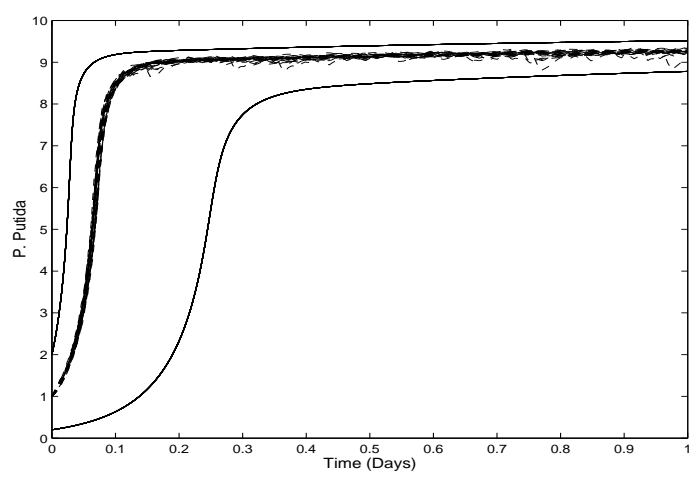

Figure 4. Concentration of Pseudomonas putida and its bounds.

Hence, for any growth function $f$, we have that:

$$
f^{-}(s)=\frac{9.43 s}{2.82+s^{2}} \leq f(s) \leq \frac{22.5 s}{3.09+s^{2}}=f^{+}(s) .
$$

We will work with the following function:

$$
f(s)=\frac{\left[15.96+\omega_{1}(t)\right] s}{2.955+\omega_{2}(t)+s}
$$

where the functions $\omega_{1}$ and $\omega_{2}$ have been constructed interpolating two sets of random data bounded by $[-6.535,6.535]$ and $[-0.135,0.135]$ respectively. In the same way, we build a function $\Delta(t)$ bounded by $[-0.2,0.2]$.

The feedback control law is built taking $D^{*}=1$ and using the function $h: \mathbb{R} \mapsto \mathbb{R}$, defined by:

$$
h(y(t))=0.85 \tanh \left(s^{*}-y(t)\right)
$$




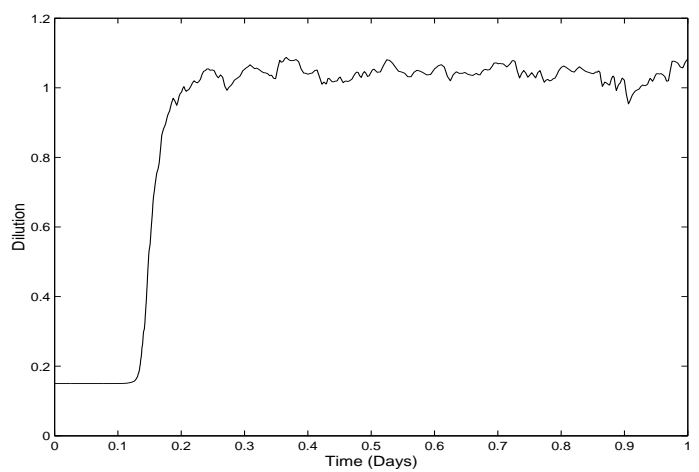

Figure 5. Dilution rate $\mathrm{v} / \mathrm{s}$ time
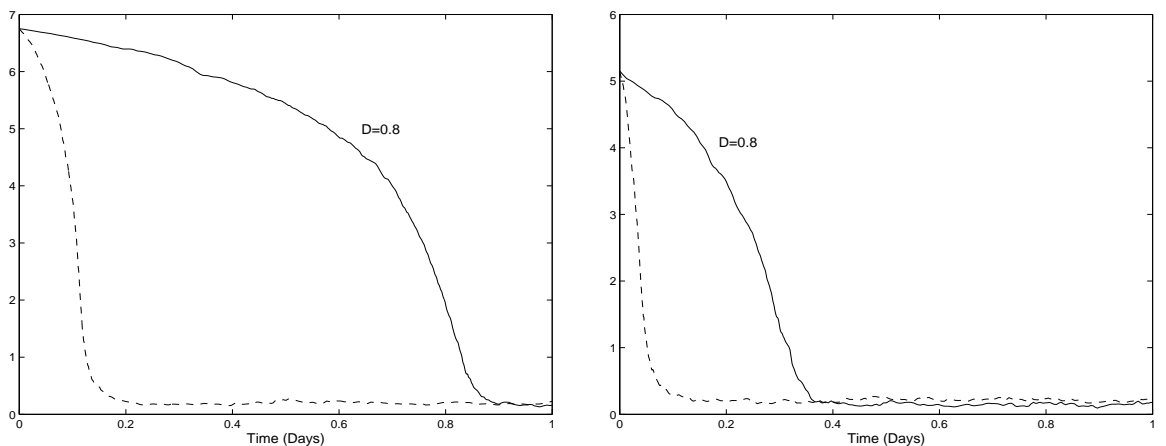

Figure 6. Substrate concentration taking initial conditions $s_{0}=$ $6.8 \mathrm{mg} / \mathrm{L}$ (left) and $s_{0}=5.1 \mathrm{mg} / \mathrm{L}$ (right): Comparison between feedback control (dashed line) and fixed dilution (continuous line).

In the numerical simulation the following parameters are considered:

$$
s_{\text {in }}=8 \mathrm{mg} / \mathrm{L} \quad s^{+}=0.49 \mu \mathrm{mg} / \mathrm{L} \quad s^{*}=0.25 \mathrm{mg} / \mathrm{L} \quad m=0.1 \mathrm{mg} / \mathrm{L} \quad \alpha=0.2 .
$$

We solve the systems using MATLAB ODE23. In order to apply Th.1, we verify easily the conditions $(\mathbf{G})$ using $f^{-}$and $f^{+}$. Figure 3 shows numerical results for the concentration of phenol graduated in $\mathrm{mg} / L$ and time axis in days. Notice that the phenol concentration becomes lower the value $s^{+}=0.49 \mathrm{mg} / L$.

Figure 4 shows the numerical results for concentration of Pseudomonas putida (graduated in $\mathrm{mg} / L)$ using ten classes of random data $\left(\omega_{1}, \omega_{2}, \Delta\right)$. Notice that this estimation could be enhanced in accuracy by using the results given in Corollary 2 and Remark 6.

We are also interested in comparing the depollution process described before with a process carried out using a fixed dilution rate (open-loop approach). Figure 6 shows simulations with two initial conditions $s_{0}$, we can see that the use of the feedback control law defined by Eq.(3) gives a faster convergence.

5.2. Simulation of marine environments. We consider Dunaniella tertiolecta growth (a chlorophilian phytoplanktonic micro-algae) in a chemostat with nitrate 
as limiting substrate [2], and realistic values (from experiments [3]) for uncertainties and parameters.

For numerical simulations we take Michaelis Menten's function defined before with the kinetic parameters $\mu_{m}$ and $k_{s}$ with experimental uncertainties given in [27] summarized in the following table (Liters are denoted by $L$, micro-atom grams by $\mu$ atg and number of cells by Cell):

\begin{tabular}{|c|c|c|}
\hline Parameter & Uncertainty & Units \\
\hline$\mu_{m}$ & $\mu_{m} \in[1.2,1.6]$ & Day $^{-1}$ \\
\hline$k_{s}$ & $k_{s} \in[0.01,0.2]$ & $\mu \mathrm{atg} / \mathrm{L}$ \\
\hline$s_{i n}$ & $s_{i n} \in[80,120]$ & $\mu$ atg/L \\
\hline$\alpha^{-1}$ & $\alpha^{-1} \in[0.15,0.6]$ & non-dimensional \\
\hline
\end{tabular}

Hence, for any growth function $f(s)$ it follows that:

$$
f^{-}(s)=\frac{1.2 s}{0.2+s} \leq f(s) \leq \frac{1.6 s}{0.01+s}=f^{+}(s) .
$$

We will work with the following function:

$$
f(s)=\frac{\left[1.4+\omega_{1}(t)\right] s}{0.105+\omega_{2}(t)+s^{2}}
$$

where $\mu_{m}=1.42, k_{s}=0.105$ and the functions $\omega_{1}$ and $\omega_{2}$ has been constructed interpolating two sets of random data bounded by $[-0.19,0.19]$ and $[-0.07,0.07]$ respectively. In the same way we build a function $\Delta(t)$ bounded by $[-0.03,0.03]$.

We will work with the following realistic values for parameters $s_{i n}, s^{*}, m$ and $\alpha$ :

$$
s_{\text {in }}=85 \mu \mathrm{gat} / \mathrm{L} \quad s^{*}=82 \mu \mathrm{gat} / \mathrm{L} \quad m=0.1 \mathrm{mg} / \mathrm{L} \quad \alpha=2 .
$$

The feedback control law is built taking $D^{*}=1.3$ and using the function $h: \mathbb{R} \mapsto$ $\mathbb{R}$ defined by:

$$
h(y(t))=1.1 \tanh \left(s^{*}-y(t)\right) .
$$

In order to apply Th.1, we verify easily the conditions (G). Figure 7 shows numerical results for the concentration of Nitrate (graduated in $\mu$ gat $L^{-1}$ and time axis in days. Figure 8 shows the numerical results for concentration of Dunaniella Tertiolecta (graduated in $\left.10^{6} \mathrm{Cell} L^{-1}\right)$ using ten classes of random data $\left(\omega_{1}, \omega_{2}, \Delta\right)$. Finally, Fig. 9 shows the dilution rate. Notice that washout is avoided, because it follows that the biomass is uniformly persistent, that means:

$$
\liminf _{t \rightarrow+\infty} x(t)>x^{-}>0 \text { for any } x(0)>0 .
$$

Persistence uniform of the biomass is essential because, as we stated in section 3 , the washout of the biomass must be avoided because the chemostat would have to be inoculated again. Notice that using a fixed dilution rate, the uniform persistence of the biomass cannot be ensured for dilution rates $D>f^{-}\left(s_{i n}\right)=1.1972$. Furthermore, taking this restriction into account, it is not possible to stabilize the substrate in a neighborhood of $s^{*}$ without control.

As before, we emphasize that the estimation given for the biomass could be enhanced in accuracy by using Corollary 2 and Remark 6 . 


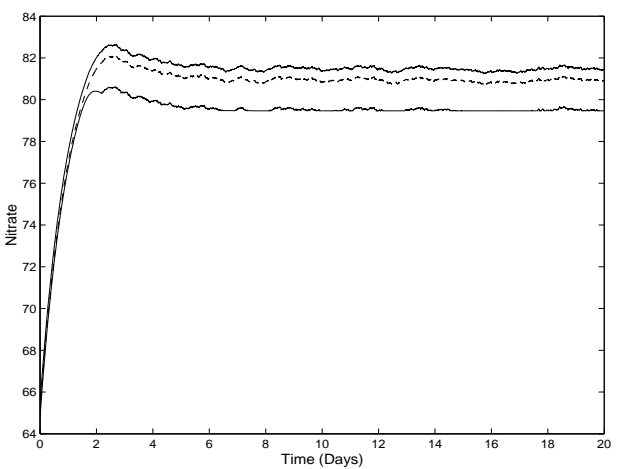

Figure 7. Concentration of nitrate (dashed line) and its bounds.

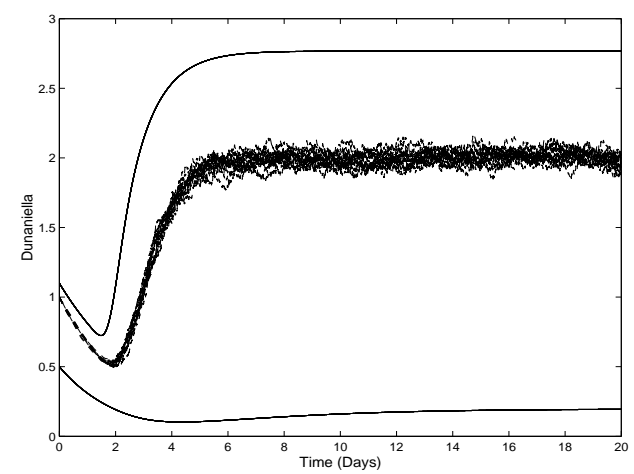

Figure 8. Concentration of Dunaniella tertiolecta and its bounds.

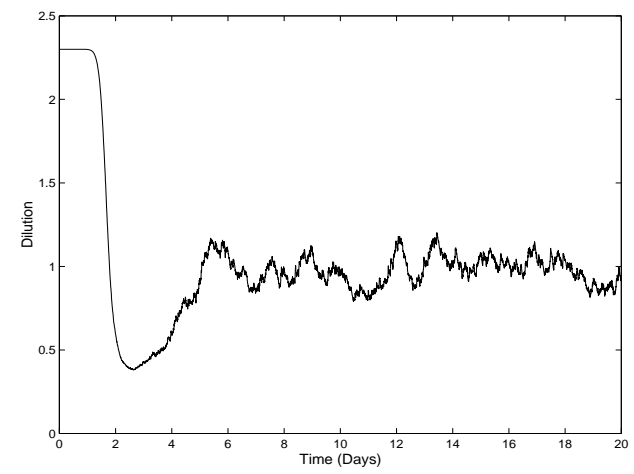

Figure 9. Dilution rate $\mathrm{v} / \mathrm{s}$ time

\section{Discussion}

The problem $(\mathcal{P})$ of feedback stabilization for a chemostat with uncertainties on its outputs and internal structure has been discussed. It has been shown that, given known bounds for the uncertainties and some technical assumptions, we are able to 
build a family of feedback control laws, which stabilize the system in a bounded set. Our approach is based on the theory of monotone dynamical systems. Furthermore, we do not assume that the uncertainties satisfy some dynamical and/or statistical properties, excluding by consequence the use of some robust control approaches given by [4] and [15].

It must be noted that, contrarily to the bibliography stated in introduction, we have considered models with mortality rate $m>0$, that makes the problem far more difficult because the "conservation principle" is not verified.

Many extensions are available in the spirit of (H1) and/or (H2): for example to suppose that the parameter $s_{i n}$ is unknown and verifies $s_{i n}^{-}(t) \leq s_{i n} \leq s_{i n}^{+}(t)$ for any $t \geq 0$ and the functions $s_{i n}^{-}, s_{i n}^{+}$are bounded and measurable.

Another natural extension of the present work would be to treat outputs of type $y(t)=s(t)+\Delta(t)$ (additive disturbance). These two extensions can be certainly solved by the methods presented in the proof of Th.1 combined with alternative/additional hypothesis.

There are some problems related but are far beyond the framework of this paper: in Remark 5 we show how a feedback control law can improve the speed of convergence toward a critical point. It will desirable to study global results and their robustness. Other related problems concern the case of competition in the chemostat, delays in some input variables (time necessary to measure the output) and state variables (delay between changes in the substrate concentration and the corresponding changes in the microorganisms).

Concluding the discussion of the control of an uncertain chemostat, we recall that other formulations are known. For example, a stabilization problem for an uncertain model of dynamics of carbon in the atmosphere is developed by Kryazhimisky and Maksimov in [16]. Inspired by game-theoretical control problems, the authors stabilize the amount of carbon in the atmosphere. It would be extremely interesting to examine this approach in our future work.

Acknowledgments: The authors would like to thank O. Bernard and L. Mailleret (INRIA-Projet COMORE) for valuable discussions. This research has been supported in part by the science and technology council of Chile (CONICYT) in the frame of INRIA-CONICYT cooperation agreement.

\section{Appendix: Planar cooperative systems}

In this appendix we present some definitions and results from the theory of planar cooperative systems [22],[23].

Definition 1. A vector field in the Euclidean space determines a cooperative system of differential equations provided that all the off-diagonal terms of its Jacobian matrix are nonnegative on a convex domain of $\mathbb{R}^{n}$.

Let us define an order in $\mathbb{R}^{2}$ by $\vec{y} \leq \vec{x}$ if $\vec{x}-\vec{y} \in \mathbb{R}_{+}^{2}$, i.e. $y_{i} \leq x_{i}$ for any $i=1,2$.

The goal is to study the asymptotic behavior of the cooperative system:

$$
\dot{x}=F(x)
$$

and to compare its solutions with these ones of the following systems:

$$
\begin{aligned}
& \dot{z}=G(z), \\
& \dot{y}=H(y)
\end{aligned}
$$

provided that the continuous functions $G, H: \Omega \mapsto \mathbb{R}^{2}$ verify $H \leq F \leq G$. 
Proposition 1 (Comparison Theorem). Assume that system (18) is cooperative. Moreover, let $x(t)$ be a solution of (18) defined on $[a, b]$, hence:

(i) If $z(t)$ is a continuous function on $[a, b]$ satisfying $(19)$ on $(a, b)$ with $z(a) \leq$ $x(a)$, then $z(t) \leq x(t)$ for all $t$ in $[a, b]$.

(ii) If $y(t)$ is a continuous function on $[a, b]$ satisfying $(20)$ on $(a, b)$ with $y(a) \geq$ $x(a)$, then $y(t) \geq x(t)$ for all $t$ in $[a, b]$.

Proof. See Theorem 3.5.1 from [23].

Proposition 2 (Asymptotic behavior). Assume that system (18) is cooperative. Moreover, if, for any initial condition $x_{0}, \omega\left(x_{0}\right)$ has a compact closure, then the solutions of system (18) are convergent to a critical point.

Proof. See Theorem 3.2.2 from [23].

\section{REFERENCES}

[1] Bastin G, Dochain D. On-line estimation and adaptive control of bioreactors, Elsevier: Amsterdam, 1990.

[2] Bernard O. Étude expérimentale et théorique de la croissance de Dunaniella Tertiolecta soumise à une limitation variable de nitrate, utilisation de la dynamique transitoire pour la conception et la validation de modèles; PhD Thesis, Université Paris VI, 1995.

[3] Bernard O, Malara G, Sciandra A. The effects of a controlled fluctuating nutrient environment on continuous cultures of phytoplankton monitored by computers; J.Exp.Mar.Biol.Ecol. 1996, 197:263-278.

[4] Byrnes CI, Delli Priscoli F, Isidori A. Output regulation of uncertain nonlinear systems, Birkhäuser: Boston, 1997

[5] Coddington E, Levinson N. Theory of ordinary differential equations, McGraw-Hill, 1955.

[6] Dochain D. (Ed.) Automatique des bioprocédés. Hermes: Paris, 2001.

[7] Gouzé JL, Rapaport A, Hadj-Sadok MZ. Interval observers for uncertain biological systems. Ecological Modelling. 2000, 133:45-56.

[8] Hadj-Sadok MZ, Gouzé JL. Estimation of uncertain models of activated sludge processes with interval observers. J.Proc. Contr. 2001, 11:299-310.

[9] Henson MA, Seborg DE (Eds.) Nonlinear process control. Prentice Hall, 1997.

[10] Hofbauer J. A unified approach to persistence. Acta.Appl.Math. 1989, 14:11-22.

[11] Hofbauer J, Sigmund K. Evolutionary games and population dynamics. Cambridge University Press: Cambridge, 1998.

[12] Keesman KJ, Stitger JD. Optimal parametric sensitivity control for the estimation of kinetic parameters in bioreactors. Math.Biosc. 2002, 179:95-111.

[13] Kieffer N, Walter E. Guaranteed parameter estimations for cooperative models. In Benvenuti L, De Santis A, Farina L (Eds.), Positive systems, Lecture Notes in control and information sciences, 294, Springer, 2003.

[14] Krasovskii NN, Subbotin AI. Game-Theoretical control problems. Springer-Verlag: NewYork, 1988.

[15] Krstić M, Deng H. Stabilization of nonlinear uncertain systems. Springer-Verlag: London 1998.

[16] Kryazhiminsky A, Maksimov V. On the exact stabilization of an uncertain dynamics. IIASA. Interim Report 03-067, 2003.

[17] Perruquetti W, Richard JP. Connecting Wasewski's conditions with M-matrices: Application to constrained stabilization. Dynamic systems and Applications. 1996,5:81-96.

[18] Raisch J, Bruce F. Modeling deterministic uncertainty. In Levine W. (Ed.), The control handbook, CRC Press, IEEE Press, 1996.

[19] Rapaport A, Dochain D. Interval observers for biochemical processes with uncertain kinetics and inputs. Math.Biosc. 2005, 193:235-253.

[20] Rapaport A, Harmand J. Robust regulation of a class of partially observed nonlinear continuous bioreactors. J.Proc. Contr. 2002, 12:291-302.

[21] Sokol W, Howell JA. Kinetics of phenol oxidation by washed cells. Biotech.Bioeng. 1981, 23:2039-2049. 
[22] Smith H, Waltman P. The theory of the chemostat. Dynamics of microbial competition. Cambridge Studies in Mathematical Biology, 13, Cambridge Univ. Press: Cambridge, 1995.

[23] Smith H. Monotone dynamical systems: an introduction to the theory of competitive and cooperative systems. Mathematical surveys and monographs, 41, AMS: Providence, 1995.

[24] Smith RJ, Wolkowicz GSK. Growth and competition in the nutrient driven self-cycling fermentation process. Canadian Appl.Math.Quart. 2003; 10:171-177.

[25] Thieme H. Convergence results and a Poincaré-Bendixson trichotomy for asymptotically autonomous differential equations. J.Math.Biol. 1992, 30:755-763.

[26] Thieme H. Asymptotically autonomous differential equations in the plane. Rock.Mount. Journ.Math. 1994, 24:351-380.

[27] Vatcheva I, Bernard O, De Jong H, Mars N. Experiment Selection for the Discrimination of Semi-Quantitative Models of Dynamical Systems. To appear in Artificial Intelligence.

E-mail address: gouze,grobledo@sophia.inria.fr

INRIA Projet COMORE, 2004 Route des Lucioles, BP 9306902 Sophia Antipolis (Cedex) France 